\title{
Cuidados vocais: questão de prevenção e saúde
}

\author{
Vocal care: question of prevention and health
}

\author{
Val eriana de Castro Guimarães ${ }^{1}$ \\ M aria A parecida do Divino Espírito Santo Reis Viana ${ }^{1}$ \\ Maria Alves Barbosa ${ }^{1}$ \\ M aria Luiza de Faria Paiva ${ }^{1}$ \\ João Antonio Gomes Tavares ${ }^{1}$ \\ Leandro Azevedo de Camargo ${ }^{1}$
}

\footnotetext{
${ }^{1}$ Hospital das Clínicas, UniversidadeFederal de Goiás. $1^{a}$ Avenida $s / n^{\circ}$, Setor Leste Universitário. 74605-020 Goiânia GO. valeriana.guimaraes@ gmail.com
}

Abstract Planned by Brazilian doctors, the $\mathrm{Na}$ tional Week of the Voice (Semana Nacional da Voz) conquered the world due to thehuge reached success. This study has the objective to demonstrate the results reached during the 9th $\mathrm{N}$ ational Week of the Voice ( 9a Semana Nacional da Voz) that took place in the H ospital das Clínicas of the Federal U niversity of Goiás. During the event, 125 patients had been selected by the phonoaudiology team and manually filled a questionnaire elaborated for the campaign in the validity of possible pharyngolaryngeal alterations. The patients had been examined by the otorhinolaryngologist using indirect laryngoscopy and, when necessary, submitted to videolaryngoscopy. After medical evaluation, it was observed that 52 people $(41,6 \%)$ had presented alterations in thespeech organs or in proximal regions, in one patient paralysis of left vocal fold was detected and one patient presented tumoral injury. Considering all the patients attended, only one presented malignant neoplasm (squamous cell carcinoma), confirmed later by biopsy.

Key words Laryngeal illnesses, Huskiness, Voice, Neoplasm, Prevention
Resumo Idealizada por médicos brasileiros, a Se mana Nacional da Voz conquistou o mundo devido ao enorme sucesso alcançado. Este estudo tem por objetivo demonstrar resultados obtidosdurante a ga Semana Nacional da Voz, realizada no H ospital das Clínicas da U niversidade Federal de Goiás (UFG). Durante 0 evento, 125 pacientes foram triados pela equipe de fonoaudiologia, preenche ram manualmente um questionário elaborado para a campanha, na vigência de possíveis alterações laringofaríngeas. Os pacientes foram examinados pelo otorrinolaringologista por meio da laringoscopia indireta e, quando necessário, submetidos à videolaringoscopia. A pós avaliação médica, observou-seque 52 pessoas ( $41,6 \%$ ) apresentaram alterações no aparelho fonador ou em regiões proximais, em um pacientefoi detectada paralisia de prega vocal esquerda eem outra lesão tumoral. Do total de pacientes atendidos, apenas um apresentou neoplasia maligna (carcinoma escamoso), confirmada posteriormente por meio de biopsia.

Palavras-chave Doenças laríngeas, Rouquidão, Voz, Neoplasia, Prevenção 
Introdução

A laringe é uma estrutura musculocartilaginosa com função específica deprodução desom nafonação, função respiratória eesfincteriana. Frequentemente acometida nas doenças que se manifestam por meio das disfonias, como em outras estruturas, a laringe é vulnerável às mais variadas enfermidades, o que se agrava mais diante de determinados fatores de risco (etilismo e tabagismo) ${ }^{1-4}$.

A voz humana é única, faz parte de nossa individualidade, e é por meio dela que podemos expressar nossos sentimentos e emoções, nos comunicar, além de ela ser instrumento de trabalho para a grande maioria da população $0^{5-10}$. Entre a população brasileira, a disfonia é a forma mais comum dos transtornos vocais. De origem multifatorial, a disfonia apresenta-se como qualquer distúrbio da voz em decorrência de uma alteração funcional e/ou orgânica do trato vocal11,12. 0 uso abusivo einadequado da voz consisteem um importante exemplo de causa de disfonia ${ }^{10}$.

A Academia Brasileira de Laringologia e Voz estima que de 20 a $30 \%$ dos brasileiros são acometidos por algum tipo de lesão nas pregas vocais. Enfermidades como essas, quando não tratadas adequadamente, podem evoluir para alte rações mais severas, como o câncer de laringe, que afeta 15 mil brasileiros por ano ${ }^{3}$.

O estado de São Paulo tem uma das mais altas prevalências de câncer laríngeo no mundo. A exposição profissional a agentes químicos e antecedentes familiares estão associados aos fatores de riscos ao câncer de laringe $\mathrm{e}^{13}$. 0 consumo de tabaco e bebidas alcoólicas ocupam papel de destaque entre os riscos para as neoplasias de cabeça e pescoço ${ }^{13-17}$.

O diagnóstico eo tratamento precoce do câncer delaringe elevam em mais de $90 \%$ as chances de sobrevida livre da doença ${ }^{3}$. Os especialistas consideram que a rouquidão persistente, pigarros, dores na garganta, dor e dificuldade ao engolir, dificuldade para respirar, cansaço vocal e perda da voz podem ser indicativos de lesões na laringe ${ }^{3,6,10}$.

A Semana Nacional da Voz chegou em 2007 ao seu nono ano. Criada por médicos brasileiros em 1999, conquistou o mundo devido ao enorme sucesso alcançado. Em 2003, o evento ganhou 0 reconhecimento das mais importantes entidades internacionais em otorrinolaringologia, como a Federação Internacional das Sociedades de Otorrinolaringologia, a Academia Americana deOtorrinolaringologia e a Sociedade Europeia de Otorrinolaringologia. 0 reconhecimento gerou a aliança responsável pela homologação do 10 Dia Mundial da Voz, 0 dia 16 de abril. A campanha da voz tem por finalidade conscientizar a população sobre a importância e os cuidados com a voz, além de orientar e prevenir as doenças do mecanismo laríngeo ${ }^{3}$.

Lamentavelmente, os hábitos de prevenção não fazem parte da cultura brasileira. Assim, 0 paciente com problemas vocais geralmente só procura o especialista quando a doença está bastante avançada.

Este estudo tem por objetivo demonstrar resultados obtidos durante a ga Semana Nacional da Voz realizada no Hospital das Clínicas da Universidade Federal de Goiás (UFG).

\section{Material e método}

Em Goiânia, a 9ạ Campanha Nacional da Voz foi comemorada pela equipe de Fonoaudiologia e Otorrinolaringologia do H ospital das Clínicas da UFG no mês de abril de 2007. No evento, foram examinadas 125 pessoas. Primeiramente, os pacientes foram triados pela equipe de fonoaudiologia da instituição, mediante aplicação de um questionário especialmente elaborado para a Campanha da Voz. 0 questionário foi preenchido manualmente para cada indivíduo pelos fonoaudiólogos da equipe, abrangendo as seguintes questões: dados de identificação, identificação de queixas vocais com múltiplas escolhas: tem voz rouca ou muito diferente dos outros? Fica rouco por mais de uma semana? Fuma? Há quanto tempo? Perde a voz? Faz uso profissional da voz? Grita muito? Fala demais? Tem voz fina (aguda) ou grossa (grave)? Pigarreia? Sente dor ou ardor na garganta? Tem amigdalites, laringites ou faringites frequentes? $\mathrm{Na}$ vigência de suspeita de possíveis al terações laringofaríngeas detectadas no momento da aplicação do questionário pelo fonoaudiólogo, o paciente era encaminhado ao otorrinolaringologista para avaliação. Em seguida, os participantes foram orientados pelo profissional, recebendo fôlder padronizado pela Campanha N acional.

Durante a avaliação médica, o paciente era submetido à laringoscopia indireta (LI), realizada com auxílio de fotóforo e espelho laríngeo. A anestesia local foi utilizada naqueles indivíduos com reflexo nauseoso mais acentuado, com xilocaína a $10 \%$. Durante o procedimento foram observadas as possíveisalterações, conformeos achados otorrinolaringológicos propostos pela $\mathrm{Cam}$ panha Nacional: exame normal, refluxo, parali- 
sia, nódulos, laringite, pólipo, cordite inespecífica, lesão tumoral, alterações estruturais mínimas, cisto, leucoplasia, edema de Reinke, papiloma, úlcera de contato, outros (especificar), benigno/suspeita tumor, hipótese diagnóstica. Para confirmação do diagnóstico em 12 indivíduos, foi realizado o exame de videolaringoscopia.

Com base nos resultados obtidos por meio dos questionários e avaliação otorrinolaringológica, foram feitas as análises dos dados.

\section{Resultados}

Dos 125 indivíduos atendidos, 101 (80,8\%) eram do gênero feminino e 25 (19,2\%) do gênero masculino, com idade de 16 a 80 anos, com média de 46,7 anos. A distribuição por faixas etárias pode ser evidenciada na Tabela 1.

As profissões variavam entre 33 tipos diferentes, sendo as mais frequentes: do lar, 51 $(40,8 \%)$; aposentados, 9 (7,2\%); estudantes, 9 $(7,2 \%)$; vendedores, $5(4 \%)$ e costureiras, $5(4 \%)$. $\mathrm{N}$ as ocupações menos frequentes, estavam três recepcionistas $(2,4 \%)$ e dois professores $(1,6 \%)$.

$\mathrm{Na}$ triagem fonoaudiológica, do total de pacientes avaliados, $29(23,2 \%)$ relataram usar sua voz profissional mente. Cento equinze indivíduos (92\%) referiram mais de quatro queixas, entre as quais: pigarros em 64 pessoas $(51,2 \%)$; dor ou ardor na garganta, 61 (48,8\%); rouquidão por mais de uma semana, 43 (34,4\%); perda da voz, $43(34,4 \%)$; voz rouca ou muito diferente dos outros, em 42 pessoas (33,6\%); amidalites, laringites ou faringites frequentes, $32(25,6 \%)$. Sete $(5,6 \%)$ pacientes relataram somente uma queixa; e três $(2,4 \%)$ não apresentaram queixa.

Os abusos vocais como gritar muito e falar demais foram relatados por 36 indivíduos
(28,8\%). Com relação ao tabagismo, 16 pacientes (12,8\%) relataram média de 23,5 anos do vício.

Pela triagem preliminar fonoaudiológica, 81 pessoas $(64,8 \%)$ apresentavam indícios de possíveis alterações vocais e/ou região orofaringolaríngea, sendo encaminhados para avaliação médica otorrinolaringológica. A pós essa avaliação, $23(30,2 \%)$ pessoas apresentaram refluxo; 11 $(14,47 \%)$ pacientes tiveram diagnóstico dedisfonia funcional; $5(6,57 \%)$ laringite; $4(5,26 \%)$ rinitealérgica; 2 (2,6\%) amigdalite crônica; 1 (1,3\%) faringite; e $1(1,3 \%)$ paciente apresentou hiperemia difusa; três (60\%) com fenda glótica; 1 (20\%) paralisia de prega vocal esquerda; e $1(20 \%)$ lesão tumoral, como mostra a Tabela 2.

Entreos 81 indivíduos encaminhadosao otorrinolaringologista, $12(14,8 \%)$ foram submetidos ao exame de videolaringoscopia para confirmação do diagnóstico.

\section{Discussão}

N o presente estudo, a população consistiu basicamente em adultos, uma vez que a maioria dos indivíduos pertencia à quarta década de vida. Dentre as profissões, a do lar foi a mais citada. As queixas mais comuns eram pigarros, dor na garganta e disfonia. Dados semelhantes foram encontrados no estudo de Ferreira e Ferreira ${ }^{4}$.

No momento da aplicação do questionário, variedades de queixas foram citadas pelos indivíduos. Esses resultados pouco diferem dos encontrados por Ferreira e Ferreira ${ }^{4}$, uma vez quea grande maioria dos indivíduos apontou numerosas queixas, não sabendo precisar qual a principal, provavelmente em razão das múltiplas escolhas presentes no formulário aplicado pelos fonoaudiólogos.

\begin{tabular}{lcc}
\hline \multicolumn{3}{l}{ Tabela 1. Distribuição de indivíduos por faixa etária. } \\
\hline $\begin{array}{c}\text { Faixa etária } \\
\text { (anos) }\end{array}$ & $\begin{array}{c}\text { Quantidade } \\
\text { de indivíduos }\end{array}$ & $\begin{array}{r}\text { Frequência } \\
\text { relativa (\%) }\end{array}$ \\
\hline $11-20$ & 03 & 2,4 \\
$21-30$ & 15 & 12 \\
$31-40$ & 26 & 20,8 \\
$41-50$ & 30 & 24 \\
$51-60$ & 33 & 26,4 \\
$61-70$ & 11 & 8,8 \\
$71-80$ & 7 & 5,6 \\
Total & 125 & 100,0 \\
\hline
\end{tabular}

\begin{tabular}{lcc}
\hline \multicolumn{1}{c}{ Tabela 2. Resultados otorrinolaringológicos. } \\
\hline \multicolumn{1}{c}{ Hipótese } & $\begin{array}{c}\text { Quantidade } \\
\text { de indivíduos }\end{array}$ & $\begin{array}{c}\text { Frequência } \\
\text { relativa (\%) }\end{array}$ \\
\hline $\begin{array}{l}\text { Ausência de alterações } \\
\text { vocais e/ou estruturas } \\
\text { orofaringolaríngeas }\end{array}$ & 73 & 58,4 \\
$\begin{array}{l}\text { Presença de alterações } \\
\text { vocais e/ou estruturas }\end{array}$ & 51 & 40,8 \\
$\begin{array}{l}\text { orofaringolaríngeas } \\
\begin{array}{l}\text { Presença de lesão tumoral } \\
\text { Total }\end{array}\end{array}$ & 01 & \\
\hline
\end{tabular}


A rouquidão persistente referida pelos indivíduos foi a queixa considerada significativa, uma vez que a persistência da rouquidão pode ser indicativa de lesões laríngeas ${ }^{3,6,10}$.

Os indivíduos tabagistas referiram estar no vício há mais de duas décadas. Estudos demonstram que o fumo se manifesta como colaborador do surgimento de lesões cancerígenas da laringe $^{13-17}$.

A grande maioria dos pacientes encaminhados pela fonoaudiologia ao ORL apresentaram alterações vocais e/ou estruturas orofaringolaríngeas. Esses achados vêm ao encontro de estudos de vários autores que demonstram a concordância entre a avaliação fonoaudiológica e a avaliação médica no diagnóstico das alterações vocais e/ou laríngeas ${ }^{2,18}$. Entretanto, tais dados contrariam os resultados de Ferreira e Ferreira ${ }^{4}$, que apontam discordância entre as opiniões dos fonoaudiólogos e os resultados observados após avaliação otorrinolaringológica.

Após avaliação otorrinolaringológica, os pacientes diagnosticados como portadores de disfonia funcional foram encaminhados à fonoterapia. Os com refluxo gastresofágico foram medicadoseencaminhados à gastroenterologia. Aqueles com alterações extralaríngeas, como laringite, amigdalite, faringite, rinite alérgica ou hiperemia difusa, receberam tratamento clínico. 0 paciente com paralisia de prega vocal esquerda foi agendado no ambulatório de otorrinolaringologia para dar seguimento ao tratamento. 0 seguimento clínico adotado nesta campanhajunto ao paciente demonstra a viabilidadee a importância decondutas como esta em eventos futuros.

0 caso mais relevanteavaliado durantea campanha apresentou, no momento do exame clíni- co, hipertrofia de amígdala direita e lesão ulcerada infiltrativa em parede lateral de orofaringe e hipofaringe, sendo retirado material para biópsia na parede lateral da orofaringe e região sublingual. 0 paciente foi encaminhado ao ambulatório de otorrinolaringologia.

$\mathrm{Na}$ semana seguinte à campanha, o paciente retornou ao ambulatório de ORL. Durante a anamnese, ele se referiu à rouquidão há dois meses, assim como tabagismo há mais de vinte anos. 0 consumo de tabaco favorece 0 surgimento de lesões neoplásicas de cabeça e pescoço ${ }^{13-17}$. O diagnóstico histopatológico de carcinoma escamoso invasor confirmou a suspeita clínica. Atualmente, o paciente encontra-se em acompanhamento com a equipe de oncologia.

\section{Consideraçõesfinais}

Das 125 pessoas atendidas na ga Campanha $\mathrm{Na}$ cional da Voz, 52 manifestaram alterações no mecanismo fonador ou estruturas proximais, e um paciente $(0,8 \%)$ apresentou neoplasia maligna (carcinoma escamoso). Todos os pacientes participantes da campanha que apresentaram alterações foram tratados e/ou agendados para consulta posterior, dando seguimento ao tratamento. 0 paciente com carcinoma foi encaminhado ao serviço de oncologia.

Um dia de campanha demonstrou a magnitude e a relevância do evento e do trabal ho entre equipes, fonoaudiologia e otorrinolaringologia, bem como a importância da participação de fonoaudiólogos com experiência na área, em campanhas como esta, assim como a importância dos cuidados vocais na prevenção dos distúrbios vocais e do câncer de laringe. 


\section{Colaboradores}

VC Guimarães trabalhou na concepção, pesquisa, metodologia ena redação final; M ADESR Viana e M A Barbosa na concepção e na redação final; M L Faria Paiva, JAG Tavares e LA Camargo trabalharam na coleta de dados.

\section{Referências}

1. Tuma J, Brasil OOC, Pontes PAL, Yasaki RK. Configuração das pregas vestibulares em laringes de pacientes com nódulo vocal. Rev Bras Otorrinolaringol 2005; 71:576-581.

2. Nemr K, Amar A, Abrahão M, Leite GCA, Köhle J, Santos AO, Corrêa LAC. Análise comparativa entre avaliação fonoaudiológica perceptivo-auditiva, análise acústica e laringoscopias indiretas para avaliação vocal em população com queixa vocal. Rev Bras Otorrinolaringol 2005; 71:13-17.

3. Sociedade Brasileira de Otorrinolaringologia. [site da Internet] [acessado 2007 abr 19]. Disponível em: http://www.sborl.com.br

4. Ferreira JB, Ferreira DS. Estudo descritivo de 451 atendimentos na Campanha da Semana Nacional da Voz. Rev Bras Otorrinolaringol 2001; 67:90-93.

5. Fortes FSG, Imamura R, Tsuji DH, Sennes LU. Perfil dos profissionais da voz com queixas vocais atendidos em um centro terciário de saúde. Rev Bras Otorrinolaringol 2007; 73:27-31.

6. Penteado RZ. Relação entre saúde e trabalho docente: percepção de professores sobre saúde vocal. Rev Soc Bras Fonoaudiol 2007; 12:18-22.

7. Fuess VL, Lorenz MC. Disfonia em professores do ensino municipal: prevalência e fatores de risco. Rev Bras Otorrinolaringol 2003; 69:807-812.

8. Gonçalves CGO, Penteado RZ, Silvério KCA. Fonoaudiologia e saúde do trabalhador: questão da saúde vocal do professor. Saúde Rev 2005; 7:45-51.

9. Penteado RZ, Bicudo-Pereira IMT. Avaliação do impacto da voz na qualidade de vida de professores. Rev Soc Bras Fonoaudiol 2003; 8:19-28.

10. Behlau M. Voz: o livro do especialista. Rio de Janeiro: Revinter; 2001.

11. Jardim R, Barreto SM, Assunção AA. Condições de trabalho, qualidade de vida e disfonia entre docentes. Cad Saude Publica 2007; 23:2439-2461.
12. Ortiz E, Costa EA, Spina AL, Crespo AN. Proposta de modelo de atendimento multidisciplinar para disfonias relacionadas ao trabalho: estudo preliminar. Rev Bras Otorrinolaringol 2004; 70:590-596.

13. Wünsch FV. The epidemiology of laryngeal cancer in Brazil. São Paulo M ed J. 2004; 122:188-194.

14. M enezes $A M B$, Horta $B L$, Oliveira $A L$, Kaufmann RAC, Duquia R, Diniz A, M otta LH, Centeno MS, Estanislau G, Gomes L. Risco de câncer de pulmão, laringe e esôfago atribuível ao fumo. Rev Saude Publica 2002; 36:129-134.

15. Goiato MC, Fernandes AUR, Santos DM, Conrado SN. Perfil dos pacientes acometidos por câncer de laringe atendidos no Centro de Oncologia BucalUnesp. Rev Odonto Ciência 2006; 21:3-8.

16. Pacella-Norman R, Urban MI, Sitas F, Cararra H. Risk factors for oesophageal, lung, oral and laryngeal cancers in black South Africans. Br J Cancer 2002; 86:1751-1756.

17. Nemr NK, Carvalho MB, Köhle J, Leite GCA, Rapoport A, Szeliga RMS. Estudo funcional da voz e da deglutição na laringectomia supracricóide. Rev Bras Otorrinolaringol 2007; 73:151-155.

18. Corazza VR, Silva VFC, Queija DS, Dedivitis RA Barros APB. Correlação entre os achados estroboscópicos, perceptivo-auditivos e acústicos em adultos sem queixa vocal. Rev Bras Otorrinolaringol 2004; 70:30-34.

Artigo apresentado em 31/01/2008

A provado em 29/10/2008

Versão final apresentada em 29/10/2008 